\title{
On Segregation during Crystal Growth from a Solution Zone
}

The distribution in a mixed crystal $\mathrm{A}_{1-x} \mathrm{~B}_{x} \mathrm{C}$ grown from an either (A, B)-rich or C-rich solution zone (e.g. travelling heater method or related techniques) is to be described by PFAN N's zone melting function which depends on the segregation coefficient and a characteristic length. Using pseudobinary mole fractions $x$ the characteristic length of the distribution function is not the actual length of the liquid solution zone but an effective zone length is to be regarded which may be considerably smaller or much bigger for C-rich or (A, B)-rich solutions zones, respectively.

Die Verteilung in einem Mischkristall $\mathbf{A}_{1-x} \mathbf{B}_{x} \mathbf{C}$, der aus einer (A, B)-reichen oder Creichen Lösungszone gezüchtet wurde (z.B. travelling heater method oder ähnliche Verfahren), ist durch Pfanss Zonenschmelzfunktion zu beschreiben, die vom Verteilungskoeffizienten und der Zonenlänge abhängt. Wenn pseudobinäre Molenbrüche $x$ zur Beschreibung verwendet werden, ist statt der tatsächlichen Länge der Lösungszone eine effektive Zonenlänge zu betrachten, die für den Verlauf der Verteilungsfunktion charakteristisch ist. Sie kann beträchtlich kleiner (C-reiche Lösungszone) oder wesentlich größer ((A,B)-reiche Lösungszone) als die wirkliche Länge der verwendeten flüssigen Zone sein.

\section{Introduction}

Besides different techniques to grow a crystal from a melt, crystal growth methods using some sort of solution have gained considerable attention with materials which cannot easily be crystallized from a stoichiometric melt. During the last twenty years, especially crystal growth from a solution zone has proved very successful in preparing a great variety of substances, because it combines the advantages of low temperature growth and steady state conditions. In fact, in steady state the dissolution of a source material just equals the crystallization from the solution zone. It is these conditions which make crystal growth from a solution zone particularly well accepted as a method of preparing mixed crystals.

The travelling heater method - sometimes better called "off-stoichiometric zone melting" - is the most employed solution zone technique in which the motion of the zone is produced by the motion of a heater. It has been successfully applied to the growth of single crystals of several semiconducting solid solutions such as ( $\mathrm{Hg}, \mathrm{Cd}$ )Te (Triboulet et al. 1985), (Cd, Zn)Te (Triboulet et al. 1983), (Hg, Zn)Te (Triboulet et al. 1986), ( $\mathrm{Pb}, \mathrm{Sn}$ ) Te (Gille et al.), (Ga, In)Sb, and (Ga, Al)As (YIP, WiLCOX).

As it is described by many authors, the principle course of PFanN's segregation function is valid for a crystal grown from a solution zone, too:

$$
c_{\mathrm{s}}(z)=c_{0}-\left(c_{0}-c_{\mathrm{L}}^{0} k\right) \exp (-k z / l) .
$$

In contrast to PfanN's original paper, the concentration of the starting molten zone $c_{\mathrm{L}}^{0}$ is allowed to differ from that of the source material $c_{0}$. (k: segregation coefficient; $z$ : axial position; $l$ : length of the liquid zone.) 
Dealing with segregation problems in mixed crystals it is usual to take the mole fraction $x$ instead of the concentration $c$ and to apply the segregation function in the same way. This is not just the right thing but a fairly good approximation because similar values of the mole volumes are one of the prerequisites which the constituents must meet to form a solid solution. Equ. (1) becomes to:

$$
x_{\mathrm{s}}(z)=x_{0}-\left(x_{0}-x_{\mathrm{L}}^{0} k\right) \exp (--k z / l) .
$$

The axial distribution of the component under investigation is characterized by an initial transition region before the concentration level is asymptotically reached which is determined by the homogeneous concentration of the feed used.

In most cases it is allowed to assume the segregation coefficient to be constant over the concentration range involved. The exponent of the segregation function strongly depends on the characteristic length which is the length of the molten zone in zone melting experiments. That is why it is usual to express the grown axial distance in terms of the relative zone length $z / l$.

It is the aim of this paper to show that it cannot be applied in the same way to crystals grown from a solution zone as several authors do. When using pseudobinary mole fractions $x$ to describe the distribution within a solid solution $\mathrm{A}_{1-x} \mathrm{~B}_{x} \mathrm{C}$ crystallized from an either (A, B)-rich or C-rich solution zone it is not correct to consider the actual length of the liquid zone to be the characteristic length of the segregation function, but the great amount of solvent is to be taken into account. For that reason a simple model is suggested which allows to calculate the characteristic length for the segregation in crystal growth from a solution zone which we call the effective zone length. It may be considerably smaller or much bigger than the actual zone length for C-rich or (A, B)-rich solutions zones, respectively.

\section{Theoretic model}

Let us consider a mixed crystal $A_{1-x} B_{x} C$ to grow from a solution zone $\left(A_{1-x_{L}} B_{x_{L}}\right)_{1-y_{L}} C_{y_{\text {L }}}$ which may be either $(\mathrm{A}, \mathrm{B})$-rich $\left(y_{\mathrm{L}}<0.5\right)$ or C-rich $\left(y_{\mathrm{L}}>0.5\right)$. It is convenient to use this pseudobinary description which better expresses that the segregation problem refers to the $\mathrm{AC} / \mathrm{BC}$ ratio (i.e. mole fraction $x$ ) whereas the deviation from stoichio-

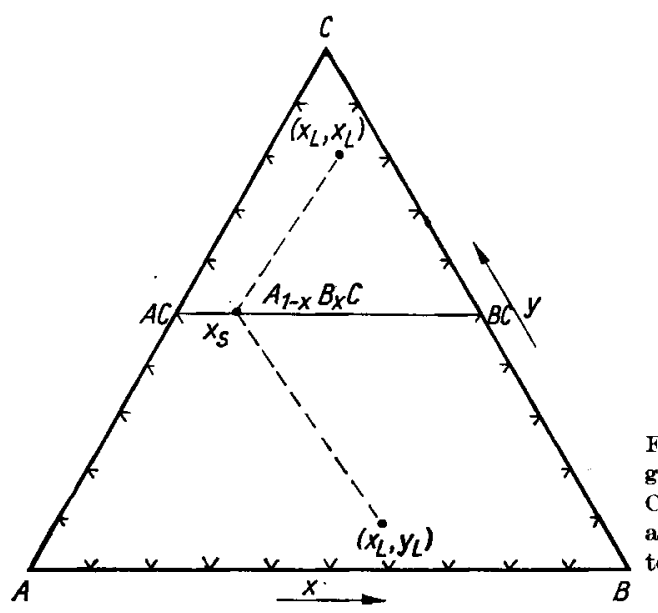

Fig. 1. Principle representation of a ternary phase diagram involving the pseudobinary solid solution $A_{1-x} B_{x}$ C. Dashed lines mark the equilibrium between a solid alloy with an $(A, B)$-rich or C-rich liquid phase (at the top read: $\left.\left(x I_{1}, y \mathrm{~L}\right)\right)$ 
metry in the liquid, which is the solubility $y_{\mathrm{L}}$, is necessary for the solution growth mechanism (Fig. 1). Prans's treatment of the segregation problem starts with the material's balance within the molten zone in terms of volume related units:

$$
\frac{d\left(c_{\mathrm{L}} \cdot l\right)}{d t}=v\left(c_{0}-c_{\mathrm{s}}\right)
$$

(c: concentration of the component under consideration in the molten zone $\left(c_{\mathbf{L}}\right)$, in the source material $\left(c_{0}\right)$, and in the grown crystal $\left(c_{\mathrm{s}}\right) ; l$ : length of the molten zone; $v$ : translation rate of the two solid/liquid phase boundaries which are assumed to be flat; $t$ : time).

Trying to write equ. (3) in terms of mole fraction $x$ we see that it is not possible $x_{\mathrm{L}}$ simply to substitute for $c_{\mathrm{L}}$, since $c_{\mathrm{L}}$ expresses the portion of the component under consideration within the ternary solution, i.e. also the solubility $y_{L}$, whereas $x_{L}$ does not. If we like to compare the pseudobinary mole fractions in the solid and liquid solution (i.e. to use the pseudobinary segregation coefficient) we have to consider the dilution of the solution zone which is the amount of solvent used. In reality $\left(c_{\mathrm{L}} \cdot l\right)$ depends on the portion of the solvent within the liquid zone. Instead of taking into account the change of $c_{\mathrm{L}}$ with varying solubility it seems more convenient to define a solubility-depending effective zone length $l_{\text {eff }}$ which allows us to describe the material's balance in terms of mole fraction $x$ for a solution zone, too:

$$
\frac{d\left(x_{\mathrm{L}} \cdot l_{\mathrm{eff}}\right)}{d t}=v\left(x_{0}-x_{\mathrm{s}}\right) \text {. }
$$

$l_{\text {eff }}$ covers all information about the actual zone length as well as the solubility $y_{L}$.

The effective zone length is that length which the liquid zone $\left(\mathrm{A}_{1-x_{L}} \mathrm{~B}_{x_{\mathrm{L}}}\right)_{1-y_{\mathrm{L}}} \mathrm{C}_{v_{\mathrm{L}}}$ would have, if it has had the same atomic fraction $y$ as the solid (i.e. $A_{1-x_{L}} B_{x_{L}}$ ). This

Crich solution zone
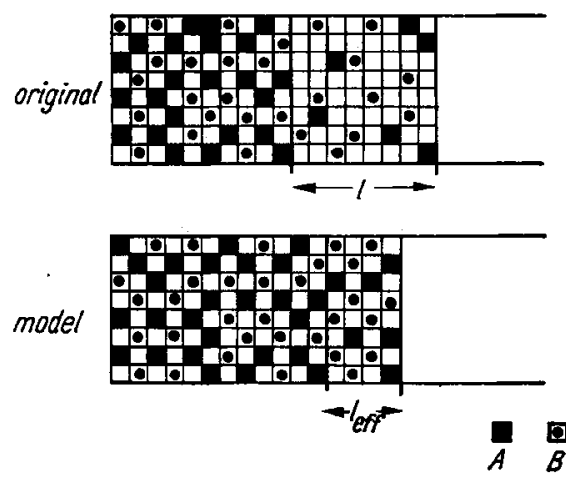

$(A, B)$-rich solution zone
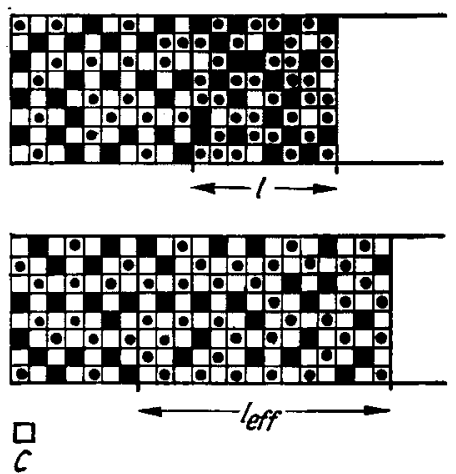

Fig. 2. Scheme of the solution zone arrangement being in equilibrium with a solid phase for a C-rich solution (on the loft) and for an $(A, B)$-rich solution (on the right). The upper drawings represent the actual liquid zones, while the lower ones represent those liquid zones which are active with respect to segregation problems and which we call the effective zone lengths $l_{\text {eff }}$

means that the actual C-rich solution zone is to be imagined to decrease its amount of $\mathrm{C}$ up to the stoichiometric composition. For this reason the effective zone length of C-rich solution zones (more general: of solution zones with a solvent which remains neutral with respect to the components to segregate) is smaller than the actual one (Fig. 2, left part). On the other hand the (A, B)-rich solution zone (a solvent which 
consists of the components to segregate) must - in mind only - be filled with $\mathrm{C}$ to reach its effective length which is bigger than in reality (Fig. 2, rigth part).

Now, these effective zone lengths allow us to compare the mole fractions in the liquid and solid phases and to apply a pseudobinary segregation coefficient $k=x_{\mathrm{g}} / x_{\mathrm{L}}$ that can be taken from the phase diagram.

To put this idea into a suitable mathematical expression it is necessary to define the following values:

$V_{\mathbf{i}} \quad$ molar volume of component $\mathbf{i}$

$x_{\mathrm{L}}, x_{\mathrm{g}}$ atomic fraction $N_{\mathrm{B}} /\left(N_{\mathrm{A}}+N_{\mathrm{B}}\right)$ of the liquid and solid phase, respectively

$y^{\mathrm{L}} \quad$ atomic fraction $N_{\mathrm{C}} /\left(N_{\mathrm{A}}+N_{\mathrm{B}}+N_{\mathrm{C}}\right)$ of the liquid phase.

For simplicity, we assume the molar volumes $V_{1}$ to be independent of temperature and the total volume of the solution to be the sum of the individual volumes of the solvent and the solute. With a $\mathrm{C}$-rich solution zone the actual zone consists of the effective (stoichiometric) zone and the excess of the pure solvent $\mathrm{C}$ :

$$
\left(A_{1-x_{\mathrm{L}}} \mathrm{B}_{x_{\mathrm{L}}}\right)_{1-y_{\mathrm{L}}} \mathrm{C}_{y_{\mathrm{L}}} \leftrightarrow\left(1-y_{\mathrm{L}}\right) \mathrm{A}_{1-x_{\mathrm{L}}} \mathrm{B}_{x_{\mathrm{L}}} \mathrm{C}+\left(2 y_{\mathrm{L}}-1\right) \mathrm{C} ; \quad y_{\mathrm{L}} \geqq 0.5 .
$$

With the constant cross section which is the prerequisite for the whole derivation of the segregation function the ratio of the effective zone length to the actual one runs to:

$$
\frac{l_{\text {eff }}}{l}=\frac{\left(1-y_{\mathrm{L}}\right)\left[\left(1-x_{\mathrm{L}}\right) V_{\mathrm{AC}}+x_{\mathrm{I}} V_{\mathrm{BC}}\right]}{\left(2 y_{\mathrm{L}}-1\right) V_{\mathrm{C}}+\left(1-y_{\mathrm{L}}\right)\left[\left(1-x_{\mathrm{L}}\right) V_{\mathrm{AC}}+x_{\mathrm{L}} V_{\mathrm{BC}}\right]} .
$$

With the approximation $V_{\mathrm{AC}} \approx V_{\mathrm{BC}}$ which is fairly well met for the most solid solution systems, equ. (5) becomes to:

$$
\frac{l_{\text {eff }}}{l} \approx \frac{1}{1+\frac{2 y_{\mathrm{L}}-1}{1-y_{\mathrm{L}}} \frac{V_{\mathrm{C}}}{V_{\mathrm{AC}}}} ; \quad y_{\mathrm{L}} \geqq 0.5
$$

In the case of crystals growing from an (A, B)-rich solution zone on the other hand, the actual liquid zone

$$
\left(\mathrm{A}_{1-x_{\mathrm{L}}} \mathrm{B}_{x_{\mathrm{L}}}\right)_{1-y_{\mathrm{L}}} \mathrm{C}_{y_{\mathrm{L}}} \leftrightarrow y_{\mathrm{L}} \mathrm{A}_{1-x_{\mathrm{L}}} \mathrm{B}_{x_{\mathrm{L}}} \mathrm{C}+\left(1-2 y_{\mathrm{L}}\right) \mathrm{A}_{1-x_{\mathrm{L}}} \mathrm{B}_{x_{\mathrm{L}}} ; \quad y_{\mathrm{L}} \leqq 0.5
$$

has to be enriched with $\left(1-2 y_{\mathrm{L}}\right) \mathrm{C}$ to take up its fictive volume according to the effective zone length. That is why, the ratio of the effective zone length to the actual one is greater than unity and runs to:

$$
\frac{l_{\mathrm{eff}}}{l}=\frac{\left(1-y_{\mathrm{L}}\right)\left[\left(1-x_{\mathrm{L}}\right) V_{\mathrm{AC}}+x_{\mathrm{L}} V_{\mathrm{BC}}\right]}{y_{\mathrm{L}}\left[\left(1-x_{\mathrm{L}}\right) V_{\mathrm{AC}}+x_{\mathrm{L}} V_{\mathrm{BC}}\right]+\left(1-2 y_{\mathrm{L}}\right)\left[\left(1-x_{\mathrm{L}}\right) V_{\mathrm{A}}+x_{\mathrm{L}} V_{\mathrm{B}}\right]} .
$$

With the above mentioned approximation, which is in general not valid for the molar volumes $V_{A}$ and $V_{B}$ of the solvent, we get:

$$
\frac{l_{\text {eff }}}{l} \approx \frac{1-y_{\mathrm{L}}}{y_{\mathrm{L}}+\frac{1-2 y_{\mathrm{L}}}{V_{\mathrm{AC}}}\left[\left(1-x_{\mathrm{L}}\right) V_{\mathrm{A}}+x_{\mathrm{L}} V_{\mathrm{B}}\right]} ; \quad y_{\mathrm{L}} \leqq 0.5 .
$$

With the effective zone lengths defined in equ. (5) and (7) for crystal growth from a C-rich or (A, B)-rich solution zone, respectively, equ. (2) changes to:

$$
x_{\mathrm{s}}(z)=x_{0}-\left(x_{0}-x_{\mathrm{L}}^{0} k\right) \exp \left(-k z / l_{\mathrm{eff}}\right)
$$

which is valid for segregation during crystal growth from a solution zone, e.g. for the travelling heater method and related techniques. 


\section{Diseussion}

Figure 3 shows how the ratio of the effective zone length to the actual one depends on the atomic fraction $y_{\mathrm{L}}$ of the liquid zone. Values are taken from the $\mathrm{Pb}-\mathrm{Sn}-\mathrm{Te}$ system. This means, $y_{\mathrm{L}}<0.5$ belongs to a $(\mathrm{Pb}, \mathrm{Sn})$-rich solution zone used for example in the ( $\mathrm{Pb}, \mathrm{Se}) \mathrm{Te}$ growth by TSM (BANSARAGTSCHIN et al.) or by THM (J̈̈GER, SEIPP).

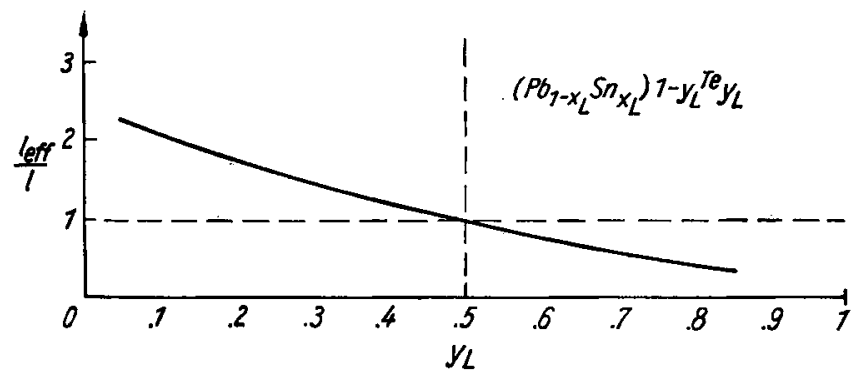

Fig. 3. Ratio of the effective $\left(l_{\mathrm{eff}}\right)$ to the actual $(l)$ zone length versus the atomic fraction $y_{L}$ of the liquid solution zone $\left(\mathrm{Pb}_{1-x_{\mathrm{L}}} \mathrm{Sn}_{x_{\mathrm{L}}}\right)_{1-\mathrm{L}} \mathrm{Te} \mathrm{y}_{\mathrm{L}}$, according to equ. (6) and (8) (read $\left.\left(\mathrm{Pb}_{1}-x_{L} \mathrm{Sn}_{x_{L}}\right) \mathrm{Te} y_{L}\right)$

With increasing solubility $y_{\mathrm{L}}$ (caused by an increasing temperature of the phase boundaries) the ratio $l_{\text {eff }} / l$ diminishs and equals unity with $y_{\mathrm{L}}=0.5$, i.e. for a stoichiometric melt. This is the original case of stoichiometric zone melting. With a further increasing tellurium concentration (i.e. with a Te-rich solution zone) the effective zone length becomes smaller than the used liquid zone. This is to be observed in THM growth of ( $\mathrm{Pb}, \mathrm{Sn})$ Te from Te-rich solutions zones (GILLE et al.) Apart from better growth conditions with Te-rich zones there is a considerable difference in the first part of the segregation functions of the two different solvents used.

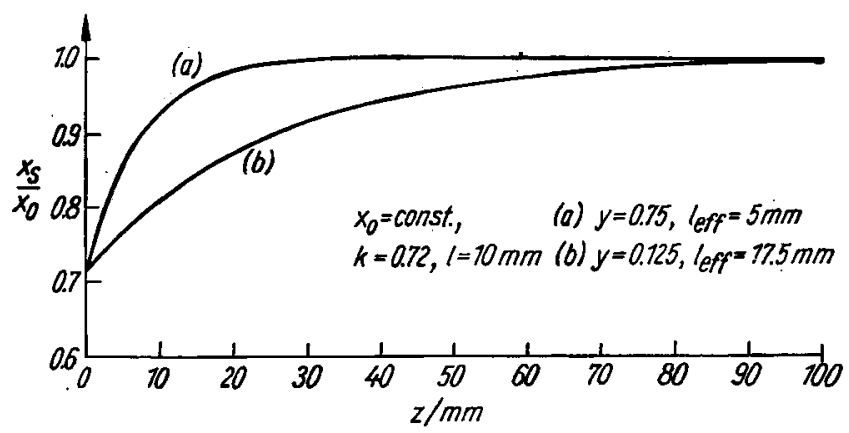

Fig. 4. Axial distribution $x_{8}$ of a mixed crystal $A_{1-x s} B_{x_{B}} C$ according to equ. (9) grown from a C-rich (curve a) or (A, B)-rich solution zone (curve b)

In Figure 4 the theoretic distribution $x_{\mathrm{s}}$ of a mixed crystal $A_{1-x_{8}} \mathbf{B}_{x_{8}} \mathrm{C}$ according to equ. (9) is pictured versus the axial position $z$ for a $\mathrm{C}$-rich solution zone (a) and an (A, B)-rich zone (b) with the actual zone length being equal in both cases. The conditions are chosen consistent with Figure 2 but differ from those of the $\mathrm{Pb}-\mathrm{Sn}-\mathrm{Te}$ system by taking the same segregation coefficients for the (A, B)-rich as well as the C-rich side to better demonstrate the great influence of the kind of solvent used. It is to be seen that with the aim of growing homogeneous crystals in solid solution systems it is much better to choose that component as the solvent which remains neutral with respect to segregation problems like in our example component $\mathrm{C}$ (i.e. $\mathrm{Te}$ for $(\mathrm{Pb}$. 
$\mathrm{Sn}) \mathrm{Te}$ ) does. It causes a relatively small effective zone length which is the characteristic length of the solution zone segregation function. Otherwise it will take the growing crystal a long distance to reach the level region of the distribution function, sometimes nearly as long as the total length of a THM-grown crystal.

Despite of all advantages of the solvent $\mathrm{C}$ of our example, it must be mentioned that this solvent acts as a relatively poor buffer when the growth conditions like the temperature or the homogeneity of the feed material are not as constant as they should be and as they are assumed in Prann's derivation.

\section{Experimental evidence}

$\mathrm{Pb}_{1-x} \mathrm{Sn}_{x}$ Te crystals were grown by the travelling heater method using $\mathrm{Te}$ as the solvent. Details of the experimental procedure have been described elsewhere (GILLE et al.; GILLE). Use was made of a monocrystalline $\mathrm{PbTe}$ seed so that the $\mathrm{Pb}_{1-x} \mathrm{Sn}_{x} \mathrm{Te}$ crystal grew with a constant cross section from the very beginning as it is supposed in the segregation function.

In Figure 5 the distribution of the mole fraction $x$ in the first part of a $\mathrm{Pb}_{1-x} \mathrm{Sn}_{x} \mathrm{Te}$ crystal is plotted versus the axial position. Mole fractions were measured by electron probe microanalysis ( $\mathrm{Sn}-\mathrm{L}_{\alpha}$ radiation) along a longitudinal section of the crystal. It is to be seen that the growing crystal reaches the original composition of the source material after a distance of about $15 \mathrm{~mm}$.
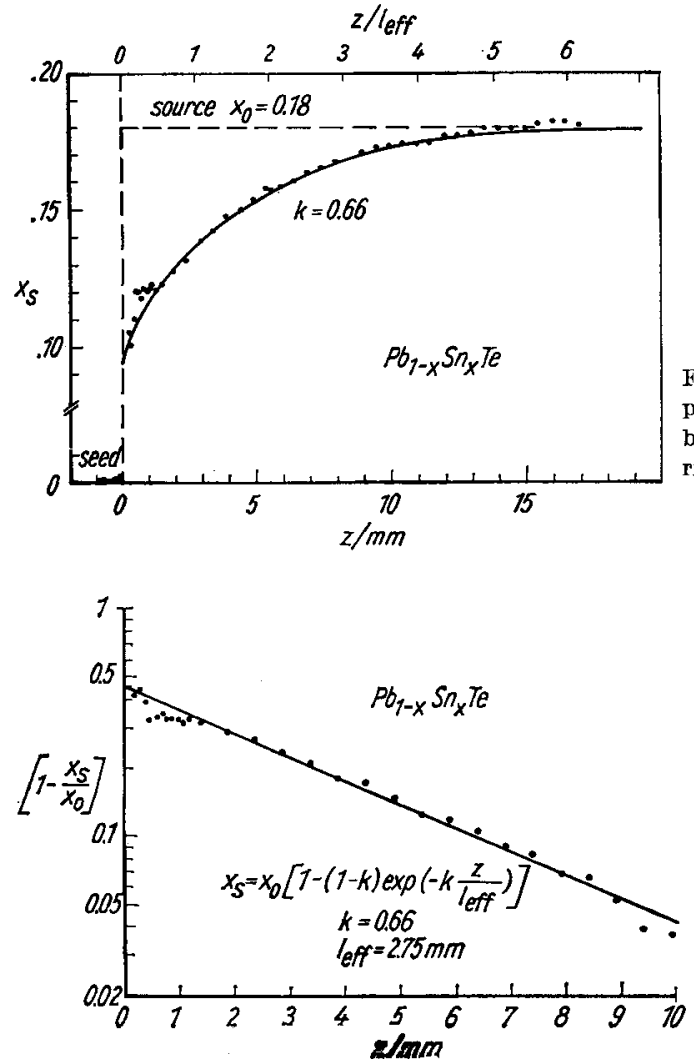

Fig. 5. Axial distribution $x_{8}$ measured by electron probe microanalysis in a $\mathrm{Pb}_{1-x} \mathrm{Sn}_{x}$ Te crystal grown by the travelling heater method (THM) from an Terich solution zone
Fig. 6. Logarithmic representation of the axial distribution $x_{\mathrm{a}}$ in a $\mathbf{P b}_{1-x} \mathrm{Sn}_{x}$ Te crystal grown by 'THM from a Te-rich solution zone (compare Fig. 5) revealing the segregation coefficient $k$ and the effective zone length $l_{\text {eff }}$ 
When the distribution of the same crystal $\left(1-x_{\mathrm{s}} / x_{0}\right)$ is pictured in a logarithmic scale versus the axial position (Fig. 6) we get a linear course with the slope $\left(-k / l_{\mathrm{eff}}\right)$. From the intersection with $z=0$, the segregation coefficient is determined to be $k=0.66$, which is in very good agreement with the data known from WAGNER and Willardoson, and Calawa et al. From this it follows an effective zone length of $l_{\text {eff }}=2.75 \mathrm{~mm}$ whereas the actual one was about $7 \mathrm{~mm}$. This is the reason for the relatively fast convergency of the distribution function which allows the growth of homogeneous mixed crystals of $(\mathrm{Pb}, \mathrm{Sn}) \mathrm{Te}$ from a Te-rich solution zone by the travelling heater method.

The author is grateful to Dr. B. Christ for carrying out the electron probe microanalysis measurements.

\section{References}

Bansaragtschin, B., Link, R., Lehmann, G.: Kristall u. Technik 13 (1978) 269

Calawa, A. R., Harman, T. C., Finn, M., Youtz, P.: Trans. Met. Soc. AIME 242 (1968) 374

Gille, P., Mühlberg, M., Parthier, L., Rudolph, P.: Cryst. Res. Technol. 19 (1984) 881

Gilte, P.: Dissertation, Humboldt-Universität Berlin 1984

JäGER, H., SeLPP, E.: Verh. DPG 1 (1978) 86

Pfann, W. G.: Trans. Met. Soc. AIME 194 (1952) 747

Triboutet, R., Nev, G., Fotouni, B.: J. Cryst. Growth 65 (1983) 262

Triboulet, R., Nguyen DuY, T., Durand, A.: J. Vac. Sci. Technol. A 3 (1985) 95

Triboulet, R., Lasbley, A., Toulouse, B., Granger, R.: J. Cryst. Growth 79 (1986) 695

Wagner, J. W., Willardson, R. K.: Trans. Met. Soc. AIME 242 (1968) 366

YIP, V. F. S., WILcox, W. R.: Mat. Res. Bull. 11 (1976) 895

(Received August 31, 1987

accepted September 12, 1987)

\section{Author's address :}

Dr. P. GILLE

Humboldt-Universität zu Berlin, Sektion Physik, Bereich Kristallographie

Invalidenstraße 110

Berlin

DDR-1040 